\title{
SPHERICAL FUNCTORS ON THE KUMMER SURFACE
}

\author{
ANDREAS KRUG AND CIARAN MEACHAN
}

\begin{abstract}
We find two natural spherical functors associated to the Kummer surface and analyze how their induced twists fit with Bridgeland's conjecture on the derived autoequivalence group of a complex algebraic K3 surface.
\end{abstract}

\section{$\S 1$. Introduction}

Let $\mathcal{D}(X)$ be the bounded derived category of coherent sheaves on a smooth complex projective variety $X$, and let $\operatorname{Aut}(\mathcal{D}(X))$ denote the set of isomorphism classes of exact $\mathbb{C}$-linear autoequivalences of $\mathcal{D}(X)$. Then we always have a subgroup $\operatorname{Aut}_{\text {st }}(\mathcal{D}(X)) \subset \operatorname{Aut}(\mathcal{D}(X))$ of standard autoequivalences which is generated by pushforwards along automorphisms, twists by line bundles, and shifts. The complement of this subgroup, if nonempty, is usually very interesting and mysterious; its elements will be called nonstandard autoequivalences.

The most successful way to construct nonstandard autoequivalences was discovered in the groundbreaking work of Seidel and Thomas [14] on spherical objects. This was extended by Huybrechts and Thomas [8] to a notion of $\mathbb{P}$-objects and further still to a theory of spherical and $\mathbb{P}$-functors (see $[13],[3],[1])$.

The first example of a series of $\mathbb{P}$-functors was constructed by Addington [1, Theorem 2] for the Hilbert scheme $X^{[n]}$ of $n$ points on a K3 surface $X$. In particular, he showed that the natural functor $F: \mathcal{D}(X) \rightarrow \mathcal{D}\left(X^{[n]}\right)$ induced by the universal ideal sheaf on $X \times X^{[n]}$ is a $\mathbb{P}^{n-1}$-functor in the sense of $[1$, Section 3] and thus gives rise to a nonstandard autoequivalence of $\mathcal{D}\left(X^{[n]}\right)$ for each $n \geq 2$. Notice that when $n=1$ this $F$ is Mukai's reflection functor (see [10, p. 362]), which coincides (up to a shift) with the spherical twist around the structure sheaf $\mathcal{O}_{X}$.

Received February 25, 2014. Revised April 10, 2014. Accepted May 12, 2014.

2010 Mathematics Subject Classification. Primary 14F05; Secondary 14J28, 18 E30. 
Inspired by this example, the second author [9, Theorem 4.1] provided the analogous result for the generalized Kummer variety $K_{n} \subset A^{[n+1]}$ associated to an abelian surface $A$. More precisely, he proved that the natural FourierMukai functor $F_{K}: \mathcal{D}(A) \rightarrow \mathcal{D}\left(K_{n}\right)$ induced by the universal ideal sheaf on $A \times K_{n}$ is again a $\mathbb{P}^{n-1}$-functor yielding a new nonstandard autoequivalence of $\mathcal{D}\left(K_{n}\right)$ for each $n \geq 2$.

This short note completes this theorem to the case $n=1$ where the generalized Kummer variety is the classical Kummer surface. The motivation to understand this particular case comes from Bridgeland's conjecture in [5, Conjecture 1.2] on the derived autoequivalence group of a complex algebraic K3 surface; roughly speaking, it says that $\operatorname{Aut}(\mathcal{D}(X))$ should be generated by standard autoequivalences and twists around spherical objects.

\section{Summary of main results}

Every abelian surface $A$ has a natural K3 surface associated to it, namely, the Kummer surface $K:=K_{1}$. It can either be defined as the blowup of the quotient $A / \iota$ along the sixteen ordinary double points, where $\iota$ denotes the involution $a \mapsto-a$ or, equivalently, as the fiber of the Albanese map $m: A^{[2]} \rightarrow A$ over zero. That is, we can identify $K$ with the subvariety of the Hilbert scheme $A^{[2]}$ consisting of those points representing length 2 subschemes of $A$ whose weighted support sums to zero. In other words, there is a universal family $\mathcal{Z} \subset A \times K$ giving rise to the commutative diagram

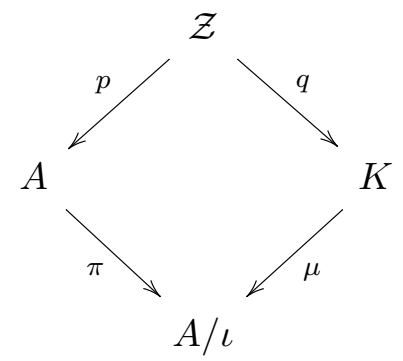

Recall that a Fourier-Mukai functor $F: \mathcal{D}(Y) \rightarrow \mathcal{D}(X)$ with left adjoint $L$ and right adjoint $R$ is said to be spherical if the cotwist $C_{F}:=$ cone(id $\stackrel{\eta}{\rightarrow}$ $R F)$ is an autoequivalence of $\mathcal{D}(Y)$ and we have a functorial isomorphism $R \simeq C L$. In particular, if $F$ is spherical, then the twist $T_{F}:=\operatorname{cone}(F R \stackrel{\epsilon}{\rightarrow}$ id $)$ is an autoequivalence of $\mathcal{D}(X)$. A spherical object $\mathcal{E} \in \mathcal{D}(X)$ corresponds to the case $F:=\left({ }_{-}\right) \otimes \mathcal{E}: \mathcal{D}(\mathrm{pt}) \rightarrow \mathcal{D}(X)$. 
In this article, we focus on the exact triangle $F \rightarrow F^{\prime} \rightarrow F^{\prime \prime}$ of FourierMukai functors $\Phi_{\mathcal{E}}: \mathcal{D}(A) \rightarrow \mathcal{D}(K)$ induced by the structure sequence of $\mathcal{Z}$ :

$$
F:=\Phi_{\mathcal{I}_{\mathcal{Z}}}, \quad F^{\prime}:=\Phi_{\mathcal{O}_{A \times K}}=\mathrm{H}^{*}\left({ }_{-}\right) \otimes \mathcal{O}_{K}, \quad F^{\prime \prime}:=\Phi_{\mathcal{O}_{\mathcal{Z}}}=q_{*} p^{*}
$$

Our main result is the following.

Theorem 1 (Proposition 2.1 and Corollary 2.4). Both $F$ and $F^{\prime \prime}$ are spherical functors with cotwists $C_{F} \simeq C_{F^{\prime \prime}} \simeq \iota^{*}$.

In light of [5, Conjecture 1.2], this immediately raises the question of whether the twists $T_{F}, T_{F^{\prime \prime}} \in \operatorname{Aut}(\mathcal{D}(K))$ associated to these functors $F, F^{\prime \prime}$ can be decomposed into twists $T_{\mathcal{E}}$ around spherical objects $\mathcal{E} \in \mathcal{D}(K)$. We answer this question with the following.

Theorem 2 (Proposition 2.1 and Corollary 2.4). The induced twists $T_{F}$, $T_{F^{\prime \prime}} \in \operatorname{Aut}(\mathcal{D}(K))$ decompose in the following way:

$$
T_{F^{\prime \prime}} \simeq \prod_{i} T_{\mathcal{O}_{E_{i}}(-1)}^{-1} \circ M_{\mathcal{O}_{K}(E / 2)}[1] \simeq \prod_{i} T_{\mathcal{O}_{E_{i}}} \circ M_{\mathcal{O}_{K}(-E / 2)}[1]
$$

and

$$
F[1] \simeq T_{\mathcal{O}_{K}} \circ F^{\prime \prime} \quad \Longrightarrow \quad T_{F} \simeq T_{\mathcal{O}_{K}} \circ T_{F^{\prime \prime}} \circ T_{\mathcal{O}_{K}}^{-1},
$$

where $E=\bigcup_{i} E_{i}$ for the exceptional curves $E_{i}$ of the Hilbert-Chow morphism $\mu$ and $M_{\mathcal{O}_{K}(E / 2)}:=\left({ }_{-}\right) \otimes \mathcal{O}_{K}(E / 2)$.

It is easy to see that the squares $T_{F}^{2}, T_{F^{\prime \prime}}^{2}$ of our twists act trivially on the cohomology of $K$ (see [1, Section 1.4]). In fact, Corollary 2.5 shows that $T_{F}^{2} \simeq T_{F^{\prime \prime}}^{2} \simeq[2]$.

In this paper we give a different proof of Corollary 2.4 from that which could have been obtained from adapting the arguments in [9]. The advantage of our approach is that it immediately provides us with the decompositions of $T_{F}$ and $T_{F^{\prime \prime}}$ as stated above.

\section{$\S 2$. Natural functors on the Kummer surface}

Another way of describing $K$ is by first blowing up the fixed points $\tilde{A} \rightarrow A$. Since the fixed points are $\iota$-invariant, the involution $\iota$ lifts to an involution 
$\tilde{\iota}$ of $\tilde{A}$ :

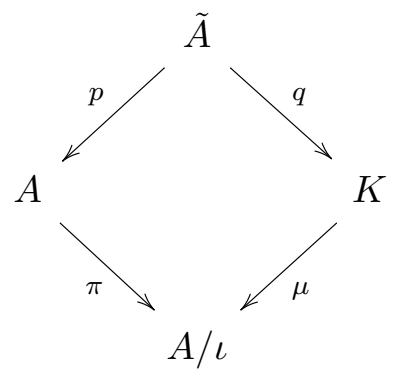

The quotient $\tilde{A} \rightarrow K$ is a double cover ramified over 16 exceptional curves $E_{i}$. Moreover, the canonical bundle formula for the blowup yields $\omega_{\tilde{A}} \simeq$ $\mathcal{O}\left(\sum \tilde{E}_{i}\right)$, where the $\tilde{E}_{i}$ are the exceptional divisors in $\tilde{A}$. Their images $E_{i}$ in $K$ satisfy $q^{*} \mathcal{O}\left(E_{i}\right) \simeq \mathcal{O}\left(2 \tilde{E}_{i}\right)$ and $q_{*} \mathcal{O}_{\tilde{A}} \simeq \mathcal{O}_{K} \oplus \mathcal{O}\left(-\frac{1}{2} \sum E_{i}\right)$. (See [7, Chapter 1.1] for more details.) We set $E:=\bigcup_{i} E_{i}$ and $\tilde{E}:=\bigcup_{i} \tilde{E}_{i}$ from now on.

Proposition 2.1. We have that $F^{\prime \prime}: \mathcal{D}(A) \rightarrow \mathcal{D}(K)$ is a spherical functor with cotwist $C_{F^{\prime \prime}} \simeq \iota^{*}$ and twist

$$
T_{F^{\prime \prime}} \simeq \prod_{i} T_{\mathcal{O}_{E_{i}}(-1)}^{-1} \circ M_{\mathcal{O}_{K}(E / 2)}[1]
$$

Proof. The pushforward along the double cover $q_{*}: \mathcal{D}(\tilde{A}) \rightarrow \mathcal{D}(K)$ is a spherical functor with cotwist $C_{q_{*}} \simeq M_{\mathcal{O}_{\tilde{A}}(\tilde{E})} \circ \tilde{\iota}^{*} \simeq S_{\tilde{A}} \circ \tilde{\iota}^{*}[-2]$ and twist $T_{q_{*}} \simeq M_{\mathcal{O}_{K}(E / 2)}[1]$ (see [1, Section 1.2, Examples 5 and 6$]$ ).

By [11, Theorem 4.3], we have a semiorthogonal decomposition

$$
\mathcal{D}(\tilde{A}) \simeq\left\langle\mathcal{O}_{\tilde{E}_{1}}(-1), \ldots, \mathcal{O}_{\tilde{E}_{16}}(-1), p^{*} \mathcal{D}(A)\right\rangle .
$$

We set $\mathcal{A}:=\left\langle\mathcal{O}_{\tilde{E}_{1}}(-1), \ldots, \mathcal{O}_{\tilde{E}_{16}}(-1)\right\rangle$ and $\mathcal{B}:=p^{*} \mathcal{D}(A)$ so that $\mathcal{D}(\tilde{A}) \simeq$ $\langle\mathcal{A}, \mathcal{B}\rangle$. Since $\mathcal{D}(\tilde{A}) \simeq\left\langle S_{\tilde{A}} \mathcal{B}, \mathcal{A}\right\rangle$ by $\left[4\right.$, Proposition 3.6] and $C_{q_{*}} \mathcal{B} \simeq S_{\tilde{A}} \mathcal{B}$, we have $\mathcal{D}(\tilde{A}) \simeq\left\langle C_{q_{*}} \mathcal{B}, \mathcal{A}\right\rangle$. Thus, by [6, Theorem 4.13], the restrictions $\left.q_{*}\right|_{\mathcal{A}}$ : $\mathcal{D}(A[2]) \rightarrow \mathcal{D}(K)$ (to the set $A[2] \subset A$ of 2 -torsion points) and $\left.q_{*}\right|_{\mathcal{B}} \simeq q_{*} p^{*}=$ : $F^{\prime \prime}: \mathcal{D}(A) \rightarrow \mathcal{D}(K)$ are spherical functors with $T_{q_{*}} \simeq T_{\left.q_{*}\right|_{\mathcal{A}}} \circ T_{\left.q_{*}\right|_{\mathcal{B}}}$. Since $q_{*} \mathcal{O}_{\tilde{E}_{i}}(-1) \simeq \mathcal{O}_{E_{i}}(-1)$, we see that $T_{\left.q_{*}\right|_{\mathcal{A}}} \simeq \prod_{i} T_{\mathcal{O}_{E_{i}}(-1)}$; hence,

$$
T_{F^{\prime \prime}} \simeq T_{\left.q_{*}\right|_{\mathcal{A}}}^{-1} \circ T_{q_{*}} \simeq \prod_{i} T_{\mathcal{O}_{E_{i}}(-1)}^{-1} \circ M_{\mathcal{O}_{K}(E / 2)}[1]
$$

Notice that the cotwist of $\left.F^{\prime \prime} \simeq q_{*}\right|_{\mathcal{B}}$ is given by $S_{A} \circ \iota^{*}[-2] \simeq \iota^{*}$. 
REMARK 2.2. We can use (1) below to rewrite this decomposition as

$$
T_{F^{\prime \prime}} \simeq \prod_{i} T_{\mathcal{O}_{E_{i}}} \circ M_{\mathcal{O}_{K}(-E / 2)}[1]
$$

LEMma 2.3. We have the following isomorphism of functors

$$
F[1] \simeq T_{\mathcal{O}_{K}} \circ F^{\prime \prime}
$$

Proof. Consider the following exact triangles of functors:

$$
\operatorname{Hom}^{*}\left(\mathcal{O}_{K}, F^{\prime \prime}\right) \otimes \mathcal{O}_{K} \rightarrow F^{\prime \prime} \rightarrow T_{\mathcal{O}_{K}} \circ F^{\prime \prime} \quad \text { and } \quad F^{\prime} \rightarrow F^{\prime \prime} \rightarrow F[1] .
$$

Then it is enough to show that $\operatorname{Hom}^{*}\left(\mathcal{O}_{K}, F^{\prime \prime}\right) \otimes \mathcal{O}_{K} \simeq F^{\prime} \simeq \mathrm{H}^{*}\left(A,{ }_{-}\right) \otimes \mathcal{O}_{K}$. In other words, it is sufficient to show that $\mathrm{H}^{*}\left(K, F^{\prime \prime}\left({ }_{-}\right)\right) \simeq \mathrm{H}^{*}\left(A,{ }_{-}\right)$, but this follows from the fact that $p$ is a blowup. Indeed, we have

$$
\begin{aligned}
\mathrm{H}^{*}\left(K, F^{\prime \prime}\left({ }_{-}\right)\right) & \simeq \mathrm{H}^{*}\left(K, q_{*} p^{*}\left({ }_{-}\right)\right) \simeq \mathrm{H}^{*}\left(\tilde{A}, p^{*}\left({ }_{-}\right)\right) \\
& \simeq \mathrm{H}^{*}\left(A, p_{*} p^{*}\left(_{-}\right)\right) \simeq \mathrm{H}^{*}\left(A,,_{-}\right) .
\end{aligned}
$$

Corollary 2.4. We have that $F: \mathcal{D}(A) \rightarrow \mathcal{D}(K)$ is a spherical functor with cotwist $C_{F} \simeq \iota^{*}$ and twist

$$
T_{F} \simeq T_{\mathcal{O}_{K}} \circ T_{F^{\prime \prime}} \circ T_{\mathcal{O}_{K}}^{-1}
$$

Proof. Recall that if $F: \mathcal{D}(Z) \rightarrow \mathcal{D}(Y)$ is a spherical functor and $\Phi:$ $\mathcal{D}(Y) \stackrel{\sim}{\rightarrow} \mathcal{D}(X)$ is an equivalence of categories, then $\Phi \circ F: \mathcal{D}(Z) \rightarrow \mathcal{D}(X)$ is also a spherical functor with the same cotwist and $T_{\Phi \circ F} \simeq \Phi \circ T_{F} \circ \Phi^{-1}$. In particular, we see immediately from Lemma 2.3 that $F$ is a spherical functor with cotwist $C_{F} \simeq \iota^{*}$ and twist

$$
T_{F} \simeq T_{F[1]} \simeq T_{\mathcal{O}_{K}} \circ T_{F^{\prime \prime}} \circ T_{\mathcal{O}_{K}}^{-1} .
$$

Corollary 2.5. The squares of the spherical twists are given by

$$
T_{F}^{2} \simeq T_{F^{\prime \prime}}^{2} \simeq[2]
$$

In particular, $T_{F}^{2}, T_{F^{\prime \prime}}^{2}$ act trivially on cohomology.

Proof. Let $j: E \rightarrow K$ denote the inclusion of the exceptional divisor. Since $E$ is smooth, we can apply [1, Section 1.2, Example 5] to see that $j_{*}: \mathcal{D}(E) \rightarrow \mathcal{D}(K)$ is spherical with cotwist $C_{j_{*}} \simeq M_{\mathcal{O}_{E}(E)}[-1] \simeq S_{E}[-2]$ and twist $T_{j_{*}} \simeq M_{\mathcal{O}_{K}(E)}$. 
Set $\mathcal{A}_{1}:=\left\langle\mathcal{O}_{E_{1}}(-1), \ldots, \mathcal{O}_{E_{16}}(-1)\right\rangle$ and $\mathcal{A}_{2}:=\mathcal{A}_{1} \otimes \mathcal{O}_{E}(1)$ to be subcategories of $\mathcal{D}(E)$. Then, by [11, Theorem 2.6], we have a semiorthogonal decomposition

$$
\mathcal{D}(E) \simeq\left\langle\mathcal{A}_{1}, \mathcal{A}_{2}\right\rangle
$$

Thus, using Kuznetsov's trick in [2, Theorem 11] (which is a special case of $\left[6\right.$, Theorem 4.13]), we see that the restriction $j_{\ell}:=\left.j_{*}\right|_{\mathcal{A}_{\ell}}: \mathcal{D}(A[2]) \rightarrow \mathcal{D}(K)$ is spherical for each $\ell=1,2$, and the twists satisfy $T_{j_{1}} \circ T_{j_{2}} \simeq T_{j_{*}}$. That is,

$$
\prod_{i} T_{\mathcal{O}_{E_{i}}(-1)} \circ \prod_{i} T_{\mathcal{O}_{E_{i}}} \simeq M_{\mathcal{O}_{K}(E)}
$$

Furthermore, we have $j_{1} \simeq M_{\mathcal{O}_{K}(E / 2)} \circ j_{2}$ since $\mathcal{O}_{E_{i}}(E / 2) \simeq \mathcal{O}_{E_{i}}(-1)$, and so

$$
T_{j_{1}} \simeq T_{M_{\mathcal{O}_{K}(E / 2)} \circ j_{2}} \simeq M_{\mathcal{O}_{K}(E / 2)} \circ T_{j_{2}} \circ M_{\mathcal{O}_{K}(-E / 2)},
$$

which, after taking inverses, equates to

$$
\prod_{i} T_{\mathcal{O}_{E_{i}}(-1)}^{-1} \circ M_{\mathcal{O}_{K}(E / 2)} \simeq M_{\mathcal{O}_{K}(E / 2)} \circ \prod_{i} T_{\mathcal{O}_{E_{i}}}^{-1}
$$

This expression allows us to reduce the formula for $T_{F^{\prime \prime}}^{2}$ in the following way:

$$
\begin{aligned}
T_{F^{\prime \prime}}^{2} & \simeq \prod_{i} T_{\mathcal{O}_{E_{i}}(-1)}^{-1} \circ M_{\mathcal{O}_{K}(E / 2)} \circ \prod_{i} T_{\mathcal{O}_{E_{i}}(-1)}^{-1} \circ M_{\mathcal{O}_{K}(E / 2)}[2] \\
& \simeq M_{\mathcal{O}_{K}(E / 2)} \circ \prod_{i} T_{\mathcal{O}_{E_{i}}}^{-1} \circ \prod_{i} T_{\mathcal{O}_{E_{i}}(-1)}^{-1} \circ M_{\mathcal{O}_{K}(E / 2)}[2] \\
& \simeq M_{\mathcal{O}_{K}(E / 2)} \circ M_{\mathcal{O}_{K}(-E)} \circ M_{\mathcal{O}_{K}(E / 2)}[2] \\
& \simeq[2]
\end{aligned}
$$

where the second and third lines follow from (2) and (1), respectively.

The fact that $T_{F}^{2} \simeq[2]$ now follows immediately from Corollary 2.4.

COROLlary 2.6. The collections $\operatorname{im} F$ and $\operatorname{im} F^{\prime \prime}$ are spanning classes for $\mathcal{D}(K)$.

Proof. For any spherical functor $F: \mathcal{D}(Y) \rightarrow \mathcal{D}(X)$, we have a natural spanning class for $\mathcal{D}(X)$ given by $\operatorname{im} F \cup(\operatorname{im} F)^{\perp} \simeq \operatorname{im} F \cup \operatorname{ker} R$ (see [1, Section 1.4]). However, in our case we have $\operatorname{ker} R=0$. Indeed, let $\mathcal{E} \in \operatorname{ker} R$. Then the defining triangle for the twist $F R(\mathcal{E}) \rightarrow \mathcal{E} \rightarrow T_{F}(\mathcal{E})$ shows that $T_{F}(\mathcal{E}) \simeq \mathcal{E}$. But by Corollary 2.5 we have $\mathcal{E} \simeq T_{F}^{2}(\mathcal{E}) \simeq \mathcal{E}[2]$, which implies that $\mathcal{E} \simeq 0$; a similar argument works for $F^{\prime \prime}$. 
REMARK 2.7. This should be contrasted to the object case where every spherical object $\mathcal{E}$ is expected to have a nonempty perpendicular $\mathcal{E}^{\perp}$ (see [12, Question 1.25]).

Acknowledgments. We thank Nick Addington and Will Donovan for helpful discussions and the Hausdorff Research Institute for Mathematics for their excellent hospitality while this work was carried out. The second author is very grateful to Arend Bayer for his consistent help and support.

The first author was supported by grant SFB/TR 45 "Periods, Moduli Spaces and Arithmetic of Algebraic Varieties" of the Deutsche Forschungsgemeinschaft. The second author was supported by Engineering and Physical Sciences Research Council Doctoral Prize research fellowship grant $\mathrm{EP} / \mathrm{K} 503034 / 1$.

\section{REFERENCES}

[1] N. Addington, New derived symmetries of some hyperkähler varieties, preprint, arXiv: 1112.0487v3 [math.AG].

[2] N. Addington and P. S. Aspinwall, Categories of massless D-branes and del Pezzo surfaces, J. High Energy Phys. 2013, 39 pp. MR 3106123.

[3] I. Anno, Weak representation of tangle categories in algebraic geometry, Ph.D. dissertation, Harvard University, Cambridge, Massachusetts, 2008. MR 2711677.

[4] A. I. Bondal and M. M. Kapranov, Representable functors, Serre functors, and reconstructions (in Russian), Izv. Ross. Akad. Nauk Ser. Mat. 53 (1989), 1183-1205, 1337; English translation in Math. USSR-Izv. 35 (1990), 519-541. MR 1039961.

[5] T. Bridgeland, Stability conditions on K3 surfaces, Duke Math. J. 141 (2008), 241291. MR 2376815. DOI 10.1215/S0012-7094-08-14122-5.

[6] D. Halpern-Leistner and I. Shipman, Autoequivalences of derived categories via geometric invariant theory, preprint, arXiv:1303.5531v1 [math.AG].

[7] D. Huybrechts, Lectures on K3 surfaces, preprint, http://www.math.uni-bonn.de/ people/huybrech/K3Global.pdf (accessed 10 June, 2015).

[8] D. Huybrechts and R. Thomas, $\mathbb{P}$-objects and autoequivalences of derived categories, Math. Res. Lett. 13 (2006), 87-98. MR 2200048. DOI 10.4310/MRL.2006.v13.n1.a7.

[9] C. Meachan, Derived autoequivalences of generalised Kummer varieties, to appear in Math. Res. Lett., preprint, arXiv:1212.5286v4 [math.AG].

[10] S. Mukai, "On the moduli space of bundles on K3 surfaces, I" in Vector Bundles on Algebraic Varieties (Bombay, 1984), Tata Inst. Fund. Res. Stud. Math. 11, Tata Inst. Fund. Res., Bombay, 1987, 341-413. MR 0893604.

[11] D. O. Orlov, Projective bundles, monoidal transformations, and derived categories of coherent sheaves (in Russian), Izv. Ross. Akad. Nauk Ser. Mat. 56 (1992), 852-862; English translation in Russian Acad. Sci. Izv. Math. 41 (1993), 133-141. MR 1208153. DOI 10.1070/IM1993v041n01ABEH002182.

[12] D. Ploog, Groups of autoequivalences of derived categories of smooth projective varieties, Ph.D. dissertation, Universität Bonn, Bonn, Germany, 2005. 
[13] R. Rouquier, "Categorification of $\mathfrak{s l}_{2}$ and braid groups" in Trends in Representation Theory of Algebras and Related Topics, Contemp. Math. 406, Amer. Math. Soc., Providence, 2006, 137-167. MR 2258045. DOI 10.1090/conm/406/07657.

[14] P. Seidel and R. Thomas, Braid group actions on derived categories of coherent sheaves, Duke Math. J. 108 (2001), 37-108. MR 1831820. DOI 10.1215/S0012-7094-01-10812-0.

Andreas Krug

Mathematisches Institut

Universität Bonn

Bonn

Germany

akrug@math . uni-bonn. de

Ciaran Meachan

School of Mathematics

University of Edinburgh

Edinburgh

Scotland

ciaran.meachan@ed.ac.uk 